\section{Dental Materials}

\title{
Chemical cleaning agents and bonding to glass-fiber posts
}

\section{Ana Paula Rodrigues Gonçalves Aline de Oliveira Ogliari Patrícia dos Santos Jardim Rafael Ratto de Moraes}

School of Dentistry, Univ Federal de Pelotas, Pelotas, RS, Brazil.
Declaration of Interests: The authors certify that they have no commercial or associative interest that represents a conflict of interest in connection with the manuscript.

Corresponding Author: Rafael Ratto de Moraes

E-mail: moraesrr@gmail.com

Submitted: Sep 11, 2012

Accepted for publication: Oct 18, 2012 Last revision: Oct 29, 2012

\begin{abstract}
The influence of chemical cleaning agents on the bond strength between resin cement and glass-fiber posts was investigated. The treatments included $10 \%$ hydrofluoric acid, 35\% phosphoric acid, 50\% hydrogen peroxide, acetone, dichloromethane, ethanol, isopropanol, and tetrahydrofuran. Flat glass-fiber epoxy substrates were exposed to the cleaners for $60 \mathrm{~s}$. Resin cement cylinders were formed on the surfaces and tested in shear. All treatments provided increased bond strength compared to untreated control specimens. All failures were interfacial. Although all agents improved the bond strength, dichloromethane and isopropanol were particularly effective.
\end{abstract}

Descriptors: Acids; Post and Core Technique; Resin Cements; Shear Strength; Solvents.

\section{Introduction}

Glass-fiber posts (GFPs) are used in restorations as an alternative to cast metal post-and-core systems. ${ }^{1}$ The elastic modulus of GFPs is similar to that of dentin, leading to a more homogeneous stress distribution compared to more rigid posts. ${ }^{2}$ The retention of GFPs in the root canal is dependent on proper adhesion between the resin cement and the intraradicular dentin and between the cement and post surface. ${ }^{3}$ Previous reports have described treatments for improving the bond between resin cements and $\mathrm{GFPs}^{4-7}$ using either physical or chemical means. Physical treatments (which generally involve abrasion using airborne particles) may enhance the bond strength to quartz and GFPs, ${ }^{5}$ but are quite aggressive and may modify the GFP morphology and interfere with the fit within the root canal, potentially influencing the long-term reliability of the restoration. Chemical treatments have the advantages of being less aggressive, lower in cost, and easier to apply. Chemical agents also clean the post surface, enhancing the interaction of the silane coupling agent with the glass fibers. However, little is known regarding the effect of cleaning agents on adhesion to GFPs. The aim of this study was to investigate the effect of several chemicals used as cleaning agents on the bonding of resin cement to glass-fiber epoxy substrates. Our hypothesis was that the use of cleaning agents would significantly increase the bond strength.

\section{Methodology}

Rectangular $(6 \times 5 \mathrm{~mm}, 2 \mathrm{~mm}$ thick $)$ glass-fiber epoxy specimens 
(same composition as that of GFPs) were obtained from Angelus (Londrina, Brazil) and used as received. The specimens were divided into groups of 20 and cleaned using 10\% hydrofluoric acid, 35\% phosphoric acid, 50\% hydrogen peroxide, acetone, dichloromethane, ethanol, isopropanol, or tetrahydrofuran. The last 5 agents were used neat. An additional set of 20 specimens was left untreated as a control. Each chemical agent was applied for $60 \mathrm{~s}$ using a microbrush, followed by rinsing for $30 \mathrm{~s}$ using an air/water spray and drying with compressed air. A $\gamma$-methacryloxypropyl trimethoxysilane coupling agent solution (Angelus) was applied to each sample and the solvent was evaporated for $20 \mathrm{~s}$ using air stream.

Specimen preparation for shear testing has been described elsewhere. ${ }^{8}$ Briefly, elastomer molds containing a cylindrical orifice $1.5 \mathrm{~mm}$ in diameter and $0.5 \mathrm{~mm}$ in height were positioned on the sample surfaces. Equal volumes of dual-cure resin cement base and catalyst paste (RelyX ARC; 3M ESPE, St. Paul, USA) were mixed for $10 \mathrm{~s}$ and used to fill the orifices. The molds were covered with a polyester strip and a glass slide and subjected to a constant and uniform 500-gf cementation load for $3 \mathrm{~min}$. This procedure was important to simulate the clinical luting procedure and ensure effective contact between the cement and substrate. The specimens were photoactivated for $40 \mathrm{~s}$ using a light-emitting diode curing unit (Radii; SDI, Bayswater, Victoria, Australia) with an irradiance of $600 \mathrm{~mW} / \mathrm{cm}^{2}$.
The specimens were stored in distilled water at $37^{\circ} \mathrm{C}$ for $24 \mathrm{~h}$. The shear bond strength was tested in a mechanical testing machine (DL500; EMIC, São José dos Pinhais, Brazil) at a crosshead speed of $0.5 \mathrm{~mm} / \mathrm{min}$ until failure. The stress was applied through a thin steel wire looped around the resin cylinder and aligned with the bonding interface. Bond strength values were registered in MPa. The results were subjected to one-way ANOVA and Fisher's LSD post hoc test $(\alpha=5 \%)$. The fractured specimens were examined under $40 \times$ magnification in a stereomicroscope to determine the failure mode.

\section{Results}

The bond strength test results are depicted in Figure 1 (power of performed statistical test $=1$ ). All of the cleaning agents increased the bond strength relative to the control group $(p<0.001)$. Treatment with dichloromethane or isopropanol resulted in significantly higher bond strength than treatment with acetone or phosphoric acid ( $p \leq 0.025)$, and samples treated with dichloromethane also had significantly higher bond strengths than those treated with hydrofluoric acid or hydrogen peroxide $(p \leq 0.027)$. The bond failures were exclusively adhesive (interfacial) in all groups, with no residual resin cement left on the post surface after debonding.

\section{Discussion}

The present results provide evidence that chemical cleaning agents increase the bond strength be-
Figure 1 - Box-and-whisker plot illustrating the results of shear bond strength tests. Horizontal solid and dashed lines are medians and means. All chemical agents provided increased bond strength compared with the control group (distinct letters indicate significant differences).

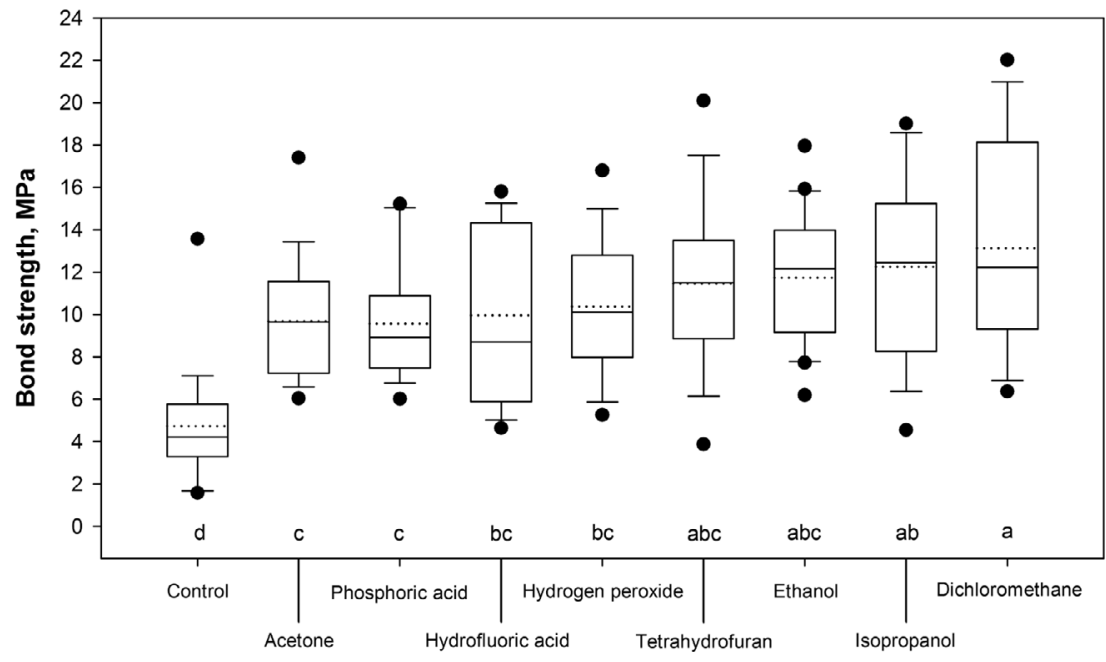

Braz Oral Res., (São Paulo) 2013 Jan-Feb;27(1):70-2 
tween resin cement and GFPs, confirming the study hypothesis. The cleaners tested were either nonaqueous organic solvents or aqueous acid/peroxide solutions. The effect of these chemicals on the post surface is potentially two-fold. On one hand, the agents remove debris left on the surface, exposing the glass fibers and improving interaction with the silane coupling agent; on the other hand, the chemicals dissolve the polymeric epoxy phase on the post surface. Although the polymer matrix is highly crosslinked and only slightly soluble, even a small amount of surface degradation could roughen the surface and enhance the mechanical keying of the resin cement. The fact that the failures were exclusively interfacial is likely due to the absence of relatively aggressive treatments (e.g. prolonged exposure times), without which adhesion occurs through shallow keying of the cement with the surface combined with chemical coupling via siloxane bonds. In this situation, there is no actual hybridization or interphase formation, and fractures tend not to be directed into the bulk of the epoxy substrate or cement but rather to concentrate at the bonding interface.

Despite the fact that some manufacturers indicate that fiber posts should be cleaned before use, there is no recommended protocol or solution for

\section{References}

1. Soares CJ, Valdivia AD, Silva GR, Santana FR, Menezes MS. Longitudinal clinical evaluation of post systems: a literature review. Braz Dent J. 2012 Apr;23(2):135-40.

2. Coelho CS, Biffi JC, Silva GR, Abrahão A, Campos RE, Soares CJ. Finite element analysis of weakened roots restored with composite resin and posts. Dent Mater J. 2009 Nov;28(6):6718.

3. Faria-e-Silva AL, Mendonça AA, Garcez RM, Oliveira AD, Moreira AG, Moraes RR. Adhesion strategy and early bond strengths of glass-fiber posts luted into root canals. Braz Oral Res. 2012 Sep-Oct;26(5):485-7.

4. Costa Dantas MC, do Prado M, Costa VS, Gaiotte MG, Simão RA, Bastian FL. Comparison between the effect of plasma and chemical treatments on fiber post surface. J Endod. 2012 Feb;38(2):215-8. this procedure, and clinicians typically clean the post surfaces using chemicals commonly available in dental practice. Compared to acid or peroxide solutions, solvents are safer and evaporate easily, and thus seem to be ideal for cleaning GFPs. The higher bond strengths obtained in samples cleaned using dichloromethane (or isopropanol in a few examples) also supports the idea of using organic solvents to clean fiber posts. Under clinical conditions, the use of cleaning agents might be expected to improve the bonding of resin luting agents to the GFPs, potentially interfering with their retention. However, there is no clinical evidence to support this statement. Other studies would be helpful in determining the effect of cleaning agents on the luting of GFPs.

\section{Conclusion}

The use of cleaning agents (particularly dichloromethane and isopropanol) improved the bonding of resin cement to glass-fiber posts.

\section{Acknowledgments}

The authors thank Angelus for the donation of the materials. The first author is grateful to the $\mathrm{Na}$ tional Council of Technological and Scientific Development (CNPq), Brazil for a scholarship (protocol 114833/2011-7).

5. Kulunk A, Kulunk T, Yenisey M. Effects of different surface pre-treatments on the bond strength of adhesive resin cement to quartz fiber post. Acta Odontol Scand. 2012 Dec;70(6):54754.

6. Monticelli F, Osorio R, Sadek FT, Radovic I, Toledano M, Ferrari M. Surface treatments for improving bond strength to prefabricated fiber posts: a literature review. Oper Dent. 2008 May-Jun;33(3):346-55.

7. Yenisey M, Kulunk S. Effects of chemical surface treatments of quartz and glass fiber posts on the retention of a composite resin. J Prosthet Dent. 2008 Jan;99(1):38-45.

8. Oliveira AS, Ramalho ES, Ogliari FA, Moraes RR. Bonding self-adhesive resin cements to glass fibre posts: to silanate or not silanate?. Int Endod J. 2011 Aug;44(8):759-63. 